\title{
ELIC: A High Luminosity And Efficient Spin Manipulation Electron-Light Ion Collider Based At CEBAF
}

\author{
Lia Merminga and Yaroslav Derbenev \\ Center for Advanced Studies of Accelerators, \\ Jefferson Laboratory, Newport News, VA 23606
}

\begin{abstract}
Electron-light ion colliders with center of mass energy between 20 and $100 \mathrm{GeV}$, luminosity between $10^{33}$ and $10^{35} \mathrm{~cm}^{-2} \mathrm{sec}^{-1}$, and polarization of both beams at or above $80 \%$ have been proposed for the study of hadronic structure. The Electron-Light Ion Collider (ELIC) facility would require the upgrade of CEBAF to 5-7 GeV energy recovering linac and the realization of an ion storage ring complex, accelerating and storing light ions of up to $150 \mathrm{GeV}$. In this report several innovative features of electron and ion beam designs and their advantages in delivering the luminosity and spin are described. These features include: electron circulator ring to reduce electron polarized source and energy recovering linac requirements, twisted spin booster and collider ring; interaction points with low beta-star and crab-crossing using the short, cooled ion bunches. Accelerator physics and technology issues for both protons/ions and electrons are presented. The feasibility of an integrated fixed target program at $25 \mathrm{GeV}$ and collider program with center of mass energy between 20 and $65 \mathrm{GeV}$ is explored.
\end{abstract}

\section{INTRODUCTION}

Thirty years after the establishment of QCD as the theory of the strong nuclear interaction, and despite significant achievements in our knowledge of the structure of hadronic matter in the intervening decades, understanding how QCD works in practice remains one of the great puzzles in nuclear physics. Some crucial questions in the field remain open and involve the role and behavior of quarks and gluons in atomic nuclei. There are still gaps in our understanding of how quarks and gluons provide the binding and the spin of the nucleon, of how quarks and gluons evolve into hadrons via the dynamics of confinement, and of how the nuclear binding arises from QCD. The nuclear physics community worldwide has suggested that a high luminosity, polarized electron-ion collider would be a powerful new microscope to probe the hadronic structure of matter, and provide answers to these questions. The luminosity of this collider should be greater than $10^{33} \mathrm{~cm}^{-2} \mathrm{sec}^{-1}$ with both beams longitudinally polarized at $80 \%$ in the interaction region. The center-of-mass energy should be variable between 20 and $100 \mathrm{GeV}$. Spin-flip of both beams is extremely desirable for exclusive measurements.

Two accelerator design scenarios are being explored for a high luminosity, high polarization collider: the scheme of colliding storage rings and the linac-ring scheme, 
with the electron beam generated from a polarized source and accelerated in a superconducting, energy recovering linac (ERL) [1]. A critical challenge of the storage ring scenario is related to the complexity of preserving and manipulating the electron spin, while the challenge of the linac-ring option stems from the necessity to generate, accelerate and energy recover a high bunch charge, high average current polarized electron beam. However, there are also significant advantages in the latter scheme, which make further exploratory studies compelling. In the linac-ring scenario, higher luminosities appear feasible and flexibility with respect to spin manipulations is greatly enhanced.

The Electron - Light Ion Collider (ELIC) facility [2] is a high luminosity, polarized electron - light ion collider in the center of mass energy range of 20-65 $\mathrm{GeV}$. It would require the upgrade of CEBAF to 5-7 GeV energy recovering linac and the realization of a storage ring complex, accelerating and storing light ions of up to $150 \mathrm{GeV}$. Preliminary design studies indicate that the luminosity of the colliding electron and light ion beams can be as high as $10^{35} \mathrm{~cm}^{-2} \mathrm{sec}^{-1}$ with an arbitrary polarization direction of either particle for up to four interaction regions. The upgrade of CEBAF could also result in a $25 \mathrm{GeV}$ fixed-target facility.

In the following, we describe the proposed CEBAF-based electron-ion collider and discuss the integration of the collider with a $25 \mathrm{GeV}$ fixed target program. We present the accelerator physics and technology challenges that must be resolved in order for the feasibility of this proposal to be established and the proposed R\&D program to address them.

\section{THE ELIC PROPOSAL}

A schematic layout of the ELIC collider is shown in Figure 1. Longitudinally polarized electrons generated from a high current polarized source are injected into the CEBAF accelerator, and accelerated to $5-7 \mathrm{GeV}$ in a single pass through the accelerator. To ease the high current polarized photoinjector and ERL requirements, a circulator ring [3] may be used. In this scenario, the electrons are injected into the circulator ring, after acceleration in the ERL, and circulate for approximately 100 revolutions while they continuously collide with the ions. They are subsequently extracted, transported back to CEBAF for deceleration and energy recovery, and are dumped at approximately their injection energy. If the polarized source development should become so advanced that the circulator ring does not offer any advantages, the electrons can still follow the same transport, but now circulate only once before being reinjected to CEBAF for energy recovery.

All ion species are injected longitudinally polarized and accelerated in a conventional ion RF linac with maximum energy of $200 \mathrm{MeV}$. Currently, two options are being considered for the ion beam stacking and acceleration after the linac. The first option comprises the conventional scheme of a 2-3 GeV kinetic energy "Figure8" [3] pre-booster ring (approximately $70 \mathrm{~m}$ in circumference, not shown in Fig. 1) followed by a large booster (coincident with the "Figure- 8 " electron circulator ring). Stripping injection of the polarized negative ions can be used to accumulate the polarized protons and deuterons in the pre-booster. However, this method is not 


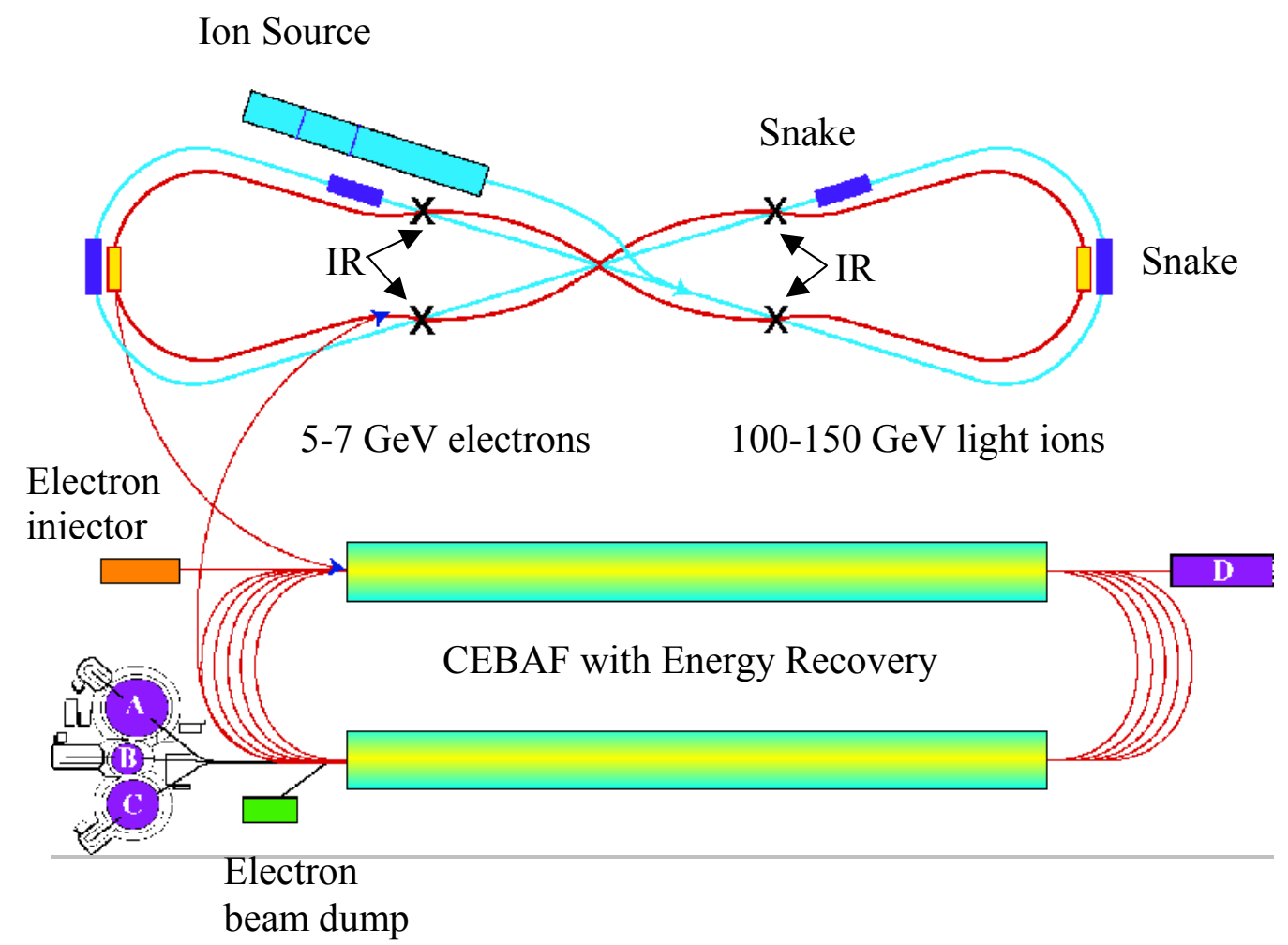

FIGURE 1. ELIC Layout.

applicable to the polarized $\mathrm{He}^{3}$ (positive ion source). To make the accumulation of polarized $\mathrm{He}^{3}$ possible, we rely on the experience of accumulation of polarized protons in the IUCF Cooler Ring with electron cooling. We consider an alternate scheme that includes a small, up to $\sim 15 \mathrm{~m}$ in circumference, $200 \mathrm{MeV}$ accumulator ring, with conventional electron cooling, following the linac and before the pre-booster. In this scheme, all ion species can be accumulated using the positive polarized sources. Another important advantage of this option is the possibility to cool the ion beam after acceleration in the linac in order to reduce the transverse emittance, if needed.

As mentioned earlier, the circulator ring can also be used as the booster ring bringing the ion energy up to $\sim 20 \mathrm{GeV}$. The ions are then injected and stored in the "Figure 8" storage ring housed in the same tunnel with the CR. "Figure- 8 " rings, including pre-booster, booster and storage ring, are used for the ions for spin preservation and flexible manipulation of all species of interest. Specifically, "Figure8 " rings have zero spin tune, as a result of which intrinsic spin resonances and spin resonance-crossing are avoided. In the "Figure-8" storage ring, longitudinal polarization for all ion species at all energies is possible by introducing solenoids in the straight sections or horizontal dipoles in the arcs. Spin rotators around the interaction points would not be needed. For protons, up to 4 simultaneous interaction regions (IRs) can exist with longitudinal or transverse polarization. Compact, nonexpensive full Siberian Snakes (shown in the Figure) can be used to provide 
longitudinal polarization for the proton beam in all 4 IRs simultaneously, while helping to stabilize the spin. For D and $\mathrm{He}^{3}$ up to 2 simultaneous IRs can exist with longitudinal or transverse spin. With "Figure- 8 " storage ring, spin-flip will work reliably without loss of polarization. To ensure that the electron spin remains longitudinal at the IRs, a Wien filter in the injector or one Siberian snake (i.e. superconducting solenoid) is required for two IRs, and a Wien filter plus 2 Siberian snakes, or three Siberian snakes without the Wien filter for 4 IRs.

A series of evolutionary upgrades to the collider facility are envisioned [2]. A first milestone may be at a luminosity of $10^{33} \mathrm{~cm}^{-2} \mathrm{sec}^{-1}$, which could be reached if the polarized electron injector current is $2.4 \mathrm{~mA}$, assuming that we use the circulator ring configuration. A next milestone, at a luminosity near $10^{34} \mathrm{~cm}^{-2} \mathrm{sec}^{-1}$ may be reached by utilizing the extremely short, of order $1 \mathrm{~cm}$, ion bunches, resulting from electron cooling [4], which allow the use of "crab crossing" [5] resulting in increase of the collision frequency and reduction of parasitic collisions. With the use of the circulator ring, the polarized electron source is required to provide about $16 \mathrm{~mA}$. From there, the maximally attainable luminosity, is obtained at the beam-beam limit of the ion ring, together with maximum collision frequency, equal to the rf frequency. Luminosity of $10^{35} \mathrm{~cm}^{-2} \mathrm{sec}^{-1}$ or greater is feasible when the ion beam energy is $100 \mathrm{GeV}$ or above.

In every stage electron cooling is introduced to suppress ion beam blowup due to IBS and its impact on the luminosity. At energies above the transition values, energy exchange between two particles at intra-beam collisions leads to horizontal emittance increase due to the energy-orbit coupling and vertical emittance increase due to $x-y$ coupling. In the limit of $\gamma>>Q$, where $Q$ is the betatron tune, and when the $\mathrm{x}-\mathrm{y}$ coupling parameter $\kappa$ is small, then the equilibrium criterion obtained by setting the IBS rate equal to the cooling rate, leads to flat beams and maximum cooling rate [4]. Thus by reducing the transverse coupling to a minimum, while conserving the beam area (which determines the luminosity), we are able to decrease the impart of IBS on luminosity. Electron cooling then leads to a flat equilibrium with large aspect ratio. Table 1 shows ELIC parameters with flat beams. Note that the beam-beam parameter has been set to the modest value of 0.01 per interaction point. The use of strong superconducting $\mathrm{rf}$, which leads to a rather high value of synchrotron tune $\left(v_{\mathrm{s}}=0.06\right)$ [4], helps stabilize the short ion bunches against microwave instabilities and beambeam interaction.

When the equilibrium described above between cooling and IBS is established, the horizontal emittance will be determined by the multiple IBS while the vertical emittance will be determined by the beam-beam interaction. The luminosity lifetime is determined by the single or Touschek scattering, which is IBS at large momentum transfer driving particles out of the core, and thus limiting the luminosity lifetime. A phenomenological model, which includes single scattering and cooling time of the scattered particles has been used to estimate an optimum set of parameters for maximum luminosity, at a given luminosity lifetime [4]. Table 1 parameters incorporate this optimization, for a luminosity lifetime of 20 hours.

Another potential limitation on the luminosity lifetime is due to the interactions between colliding beams. If the electron scatters elastically from the oncoming proton at sufficiently large angles, both of these particles are lost from the acceleration cycle. 
We evaluated this effect assuming the value of critical angle to be $0.3 \mathrm{mrad}$ (which is the design angular spread at the interaction point), and found the proton beam half-life to be 5 days for $5 \mathrm{GeV}$ electron on $50 \mathrm{GeV}$ proton beams, and 20 days for $100 \mathrm{GeV}$ protons. Contributions from inelastic processes, including electron collisions with light ions, appear to have an effect of a factor of $\sim 10$ smaller.

The same electron accelerator that is used in the collider mode can also provide up to $25 \mathrm{GeV}$ electrons for fixed target experiments for physics. This scheme would require the implementation of a 5-pass recirculator at $5 \mathrm{GeV}$ per pass as in present CEBAF. It is a subject of further investigation whether the collider and fixed target modes could run simultaneously or in alternating modes. The emittance growth due to synchrotron radiation in the CEBAF arcs at the higher energies has been addressed. A novel optics design for the higher arcs results in reduction in emittance growth by a factor of 10 compared to the present optics. The beta functions in arc 9 are $\sim 70 \mathrm{~m}$ and the spot sizes at the IP are between $0.3-0.5 \mathrm{~mm}$ at $25 \mathrm{GeV}$ [6].

The ELIC proposal is at an early stage of development. A number of technical challenges must be resolved, and several R\&D projects have been started. These include development of a high average current polarized electron source with a high bunch charge, electron cooling of protons/ions, energy recovery at high current and high energy, and the design of an interaction region and detector that support the combination of high luminosity and high detector acceptance and resolution, which are essential to carry out the proposed physics program.

This work was supported by the U.S. Department of Energy under Contract No DEAC05-84ER40150.

TABLE 1. ELIC Parameters With Flat Beams.

\begin{tabular}{lcc}
\hline Parameter & Unit & Value (Protons/Electrons) \\
\hline Beam energy & $\mathrm{GeV}$ & $150 / 7$ \\
Energy of cooling beam & $\mathrm{MeV}$ & 75 \\
Bunch rep rate & $\mathrm{GHz}$ & 1.5 \\
Particles/bunch & $10^{10}$ & $0.2 / 1$ \\
Beam current & $\mathrm{A}$ & $0.5 / 2.5$ \\
Cooling current & $\mathrm{A}$ & 2.5 \\
Horizontal emittance (norm, rms) & $\mu \mathrm{m}$ & $1 / 100$ \\
Vertical emittance (norm, rms) & $\mu \mathrm{m}$ & $0.01 / 1$ \\
Number of interaction points & & 4 \\
Total beam-beam tune shift & & $0.04 / 0.16$ \\
Laslett's tune shift in p-beam & $\mathrm{cm}^{-2} \mathrm{~s}^{-1}$ & 0.02 \\
Luminosity overall IP (10 35 ) & $\mathrm{min}^{35}$ & 2 \\
Cooling/IBS time in p-beam core & $\mathrm{h}$ & 5 \\
Luminosity Touschek's lifetime & & 20 \\
\hline
\end{tabular}

\section{REFERENCES}

1. Merminga, L., Douglas, D.R., and Krafft, G.A., Annu. Rev. Nucl. Part. Sci. 53:387-429 (2003).

2. Merminga, L., et al., Proc. European Particle Accelerator Conference (2002).

3. Derbenev, Ya., Proc. European Particle Accelerator Conference (2002)

4. Derbenev, Ya., Proc. COOL'03 Workshop (2003).

5. Derbenev, Ya., Proc. Particle Accelerator Conference (2003)

6. Chao, Y.-C. JLab Technical Note TN-99-037. 